\title{
Identification of a Congenital Dysthrombin,
}

\section{Thrombin Quick}

\author{
Ruth Ann Henriksen and Whyte G. Owen, Departments of Pathology and \\ Biochemistry, University of Iowa, Iowa City, Iowa 52240 \\ Michael E. Nesheim and Kenneth G. Mann, Department of Medicine, \\ Mayo Clinic, Rochester, Minnesota
}

\begin{abstract}
A B S T RACT A dysprothrombin designated prothrombin Quick, is isolated from the plasma of an individual with $<2 \%$ of normal functional prothrombin activity and $34 \%$ of the normal prothrombin level by immunologic assay. With Factor Xa or taipan snake venom as activators, a fragmentation pattern identical to that of normal prothrombin is observed on gel electrophoresis in dodecylsulfate. This evidence combined with the observed barium citrate adsorption of prothrombin Quick and the low activity suggests that the defect in prothrombin Quick is in the thrombin portion of the molecule. Thrombin Quick is isolated and comigrates with thrombin on dodecyl sulfate gel electrophoresis, either reduced or nonreduced. The activity of thrombin Quick on several biological substrates of thrombin is investigated. Relative to normal thrombin, thrombin Quick is $1 / 200$ as active on fibrinogen and $1 / 20-1 / 50$ as effective in activating Factors $\mathrm{V}$ and VIII and aggregating platelets. A complex with antithrombin III is detected by dodecyl sulfate gel electrophoresis. Further investigation with the active site titrant, dansylarginine- $N$-(3-ethyl-1,5-pentanediyl)amide showed that the thrombin Quick preparation has the same affinity for the titrant as thrombin, but apparently only $40 \%$ active sites per mole protein are titrable.
\end{abstract}

\section{INTRODUCTION}

Dysprothrombinemia is a rarely described blood coagulation disorder in which the functional level of pro-

Preliminary reports of this work have been presented to the American Society of Hematology, New Orleans, La., December 1978, and to the International Congress on Thrombosis and Haemostasis, London, England, July 1979 (1979. Thromb. Haemostasis. 42: 57. Abstr. 125).

Mr. Henriksen was supported by National Institutes of Health grant HL 22471-01, Dr. Nesheim by Blood Banking and Hemostasis Training Grant HL-07069, and Dr. Owen by National Institutes of Health Career Development Award HL 00348-03. Dr. Mann is an American Heart Established Investigator.

Received for publication 20 February 1980 and in revised form 8 July 1980. thrombin is decreased relative to the immunologically detectable prothrombin. This defect has been identified in nine pedigrees (1-9). Prothrombin $(72,000$ dalton) is the zymogen of the serine protease thrombin ( 37,000 dalton), which acts on fibrinogen in the final stages of blood coagulation (10). The activation reaction, catalyzed by the serine protease Factor $\mathrm{Xa}$, results in the hydrolysis of two peptide bonds, and the loss of a large activation peptide, fragment $1-2$ (35,000 dalton) from the amino terminus. The activation peptide itself plays an important role in enhancing the rate of activation (11). Because of the complex nature of prothrombin's activation, a functional dysprothrombinemia may result from a defect at several different sites in the molecule: in the fragment region, at a residue adjacent to a Factor Xa cleavage site, or in the thrombin portion of the molecule. In the present study, the dysprothrombin prothrombin Quick was isolated from the plasma of a patient originally studied by Quick $(12,13)$. The prothrombin level in this plasma assayed by a two stage method using thromboplastin or a one stage taipan venom assay is $<2 \%$ of normal. Although Quick correctly identified this patient as prothrombin deficient, only more recently has a dysprothrombin been identified by immunologic assay (6). The results reported here indicate that prothrombin Quick is hydrolyzed normally by prothrombin activators. The resultant dysthrombin, thrombin Quick has been investigated in several reactions known to occur with normal thrombin. Previously studied dysprothrombins have been defective in the fragment region or cleavage sites $(1-5,7-9)$ as determined by formation of predicted amounts of thrombin on activation by nonphysiologic activators. Thrombin Quick is unique as a genetically defective thrombin.

\section{METHODS}

Plasma from the dysprothrombinemic patient V.A. $(12,13)$, was collected into acid citrate dextrose anticoagulant by a standard double plasmapheresis technique. Approval of the University of Iowa Human Use Committee and informed 
consent of the patient were obtained. Plasma was either frozen immediately or recentrifuged to remove cellular material and stored at $-75^{\circ} \mathrm{C}$. Since prothrombin Quick was shown previously to be adsorbed by barium citrate $(6)$, a standard prothrombin isolation procedure, modified from that of Aronson (14), was used to isolate both prothrombin Quick and normal human prothrombin. For studies of thrombin, or thrombin Quick, the respective prothrombins were activated with taipan snake venom, phospholipid, and $\mathrm{Ca}^{2+}$ as activator Thrombin was isolated by a modification of the procedure originally described by Lundblad (15). The activation mixture was applied to sulfopropyl Sephadex equilibrated in $0.1 \mathrm{M}$ $\mathrm{NaCl}, 0.05 \mathrm{M}$ Tris, pH 7.5. After elution of the break-through peak, thrombin was eluted by increasing $\mathrm{NaCl}$ and stored in aliquots at $-75^{\circ}$. The thrombin and thrombin Quick concentrations were determined from the $\mathbf{A}_{\mathbf{2 8 0}}$, corrected for light scattering at $320 \mathrm{~nm}$, an $\mathrm{E}_{1 \mathrm{~cm}}^{1 \%}=16.2(16)$, assumed the same for both proteins, and $\mathrm{mol} \mathrm{wt}=37,000$. A lyophilized preparation of bovine factor $\mathrm{Xa}$ containing bovine serum albumin and Tris-maleate was a gift from C. M. Jackson, St. Louis, Mo. Phospholipid (rabbit brain cephalin), taipan snake venom, and bovine serum albumin were obtained from Sigma Chemical Co., St. Louis, Mo. Dodecyl sulfate gel electrophoresis in $10 \%$ polyacrylamide was performed as described by Laemmli (17).

Bovine Factor $V$ was prepared and assayed as described (18). Human Factor VIII was prepared by the method of Wagner et al. (19). Factor VIII was assayed by the one-stage method of Hardisty and MacPherson (20).

Platelets from a normal donor were isolated by gel filtration on Sepharose 2B (21). Equimolar $\mathrm{SrCl}_{2}$ replaced $\mathrm{MgCl}_{2}$ in the eluant that contained $0.35 \%$ bovine serum albumin (22). Platelet aggregation was studied by the method of Born and Cross (23). All aggregation studies were completed within 3.5 $h$ of venipuncture.

Fibrinogen was isolated by the procedure of Straughn and Wagner (24). To study complex formation, antithrombin III isolated as described (25), was added to thrombin or thrombin Quick and incubated for 8 min before addition of dodecyl sulfate for electrophoresis, which was performed immediately.

For the study of the presence of an active site, the competitive inhibitor dansylarginine- $N$-(3-ethyl-1,5-pentanediyl)amide (DAPA) ${ }^{1}$ was prepared as described (26). Fluorescence spectra were collected with a Hitachi-Perkin Elmer fluorescence spectrophotometer, model MPF-2A, thermostated at $25^{\circ} \mathrm{C}$.

Thrombin solutions in $0.1 \mathrm{M} \mathrm{NaCl}, 0.05 \mathrm{M}$ Tris, $\mathrm{pH} 7.5$ were used for titration. DAPA was dissolved in distilled $\mathrm{H}_{2} \mathrm{O}$ and the concentration determined from the $A_{335}$ and $\epsilon_{335}=4.01 \times 10^{3}$.

DAPA was added to the protein by using a Hamilton syringe with repeating dispensor (Hamilton Co., Reno, Nev.). After each addition, excitation spectra were obtained from 270-360 $\mathrm{nm}$, with emission detected at $550 \mathrm{~nm}$. Total titrant volume did not exceed $2 \%$ of total volume of protein solution. Fluorescence after each addition of DAPA was determined from the recorder tracing. Fluorescence for DAPA added to buffer only was also determined. The least squares regression line for the latter titration was determined and used to correct the protein fluorescence at each DAPA concentration. The corrected titration data were used to determine the respective dissociation constants and mole fraction of active sites $(n)$ as described by Gutfreund (27). The maximum fluorescence was determined by iteration to maximize the correlation coefficient as described by Nesheim et al. (26).

In each experiment described, the action of thrombin and thrombin Quick were compared in parallel with the same reagents.

${ }^{1}$ Abbreviation used in this paper: DAPA, dansylarginine$\mathrm{N}$-(3-ethyl-1,5-pentanediyl)amide.

\section{RESULTS}

To establish whether prothrombin Quick was cleaved by prothrombin activators, parallel activations of prothrombin and prothrombin Quick were analyzed by gel electrophoresis in dodecyl sulfate. The results of the activation with Factor Xa, phospholipid, and $\mathrm{Ca}^{2+}$ are shown in Fig. 1. The same set of activation fragments are observed with both proteins, and similar results were obtained with taipan venom, phospholipid, and $\mathrm{Ca}^{2+}$ as activator. These results indicate that prothrombin Quick is properly cleaved and suggest that the defect in this molecule lies in the thrombin portion of the molecule.

To investigate the properties of thrombin Quick, a preparative scale activation was performed and thrombin Quick was isolated by sulfopropyl Sephadex chromatography. This material was subjected to dodecylsulfate gel electrophoresis and was found to comigrate with thrombin and thrombin heavy chain on unreduced and reduced gels, respectively. Thus, at the level of sensitivity of this technique no difference is seen between thrombin and thrombin Quick.

Preliminary results indicated that thrombin Quick possesses significant esterase activity with benzoylarginine ethyl ester as substrate (unpublished observation). Since, the plasma prothrombin in this patient was assayed at $<2 \%$, an examination of the activity of thrombin Quick on biological substrates was of interest, particularly in view of the fact that a role for proteolytic

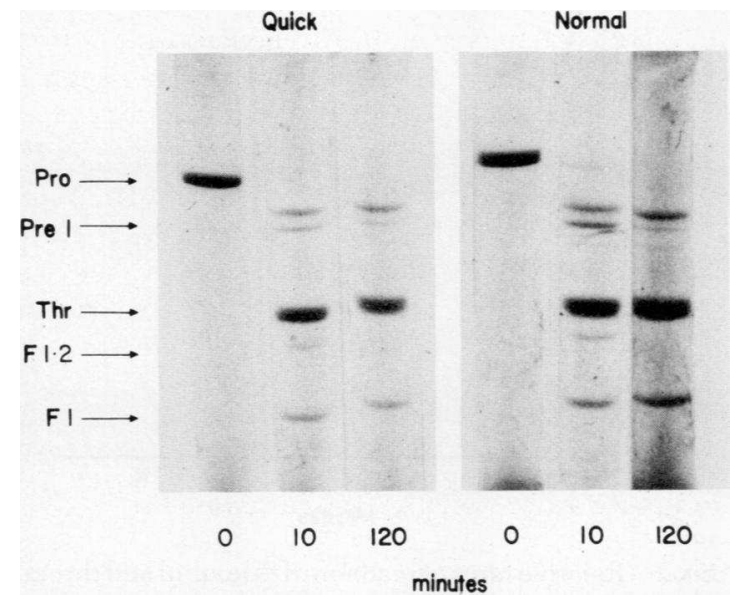

FIGURE 1 Activation of prothrombin Quick and prothrombin by Factor $\mathrm{Xa}$, phospholipid, and $\mathrm{Ca}^{2+}$. Prothrombin Quick, $66 \mu \mathrm{g} / \mathrm{ml}$ or prothrombin $77 \mu \mathrm{g} / \mathrm{ml}$; $\mathrm{Ca}^{2+}, 7 \mathrm{mM}$; Factor $\mathrm{Xa}, \sim 7 \mathrm{U} / \mathrm{ml}(30)$; and phospholipid were incubated at $22^{\circ} \mathrm{C}$. Aliquots were removed at the indicated times and the reaction stopped by addition of dodecyl sulfate at $100^{\circ} \mathrm{C}$. Gels were loaded with $5 \mu \mathrm{g}$ (prothrombin Quick) or $6 \mu \mathrm{g}$ (prothrombin) protein. The additional band seen in the 10- and 120-min gels is due to albumin in the $\mathrm{Xa}$ preparation. Pro, prothrombin; Pre 1, prethrombin 1; Thr, Thrombin; F1.2, fragment $1 \cdot 2$; F1, fragment 1 . 
catalysis has not been rigorously established in all of these reactions. In addition to the conversion of fibrinogen to fibrin in the final stage of blood coagulation, thrombin also participates in other reactions associated with blood coagulation, including the activation of Factors $\mathrm{V}$ and VIII, the aggregation of platelets, and the formation of a complex with the plasma protein inhibitor antithrombin III. The behavior of thrombin Quick in these reactions was investigated.

To determine the action of thrombin Quick on fibrinogen, dilutions of normal thrombin were made to extend the clotting time to that observed with the undiluted stock solution of thrombin Quick. Based on protein concentrations, the results indicated that thrombin Quick was 1/200 as effective as thrombin in clotting fibrinogen. Fig. 2 shows the relative initial rates of disappearance of Factor $\mathrm{V}$ (determined by appearance of Factor $\mathrm{Va}$ ) in reactions with thrombin and thrombin Quick. Relative slopes were determined from corresponding initial time points and indicate that the activation of Factor $\mathrm{V}$ by thrombin Quick occurs at $1 / 20$ the rate observed with thrombin. Thrombin Quick activation was found to deviate from linearity in the first order plot (Fig. 2) after about half the total Factor $\mathrm{V}$ had been activated (figure shows rates normalized to equivalent protein concentrations for both enzymes).

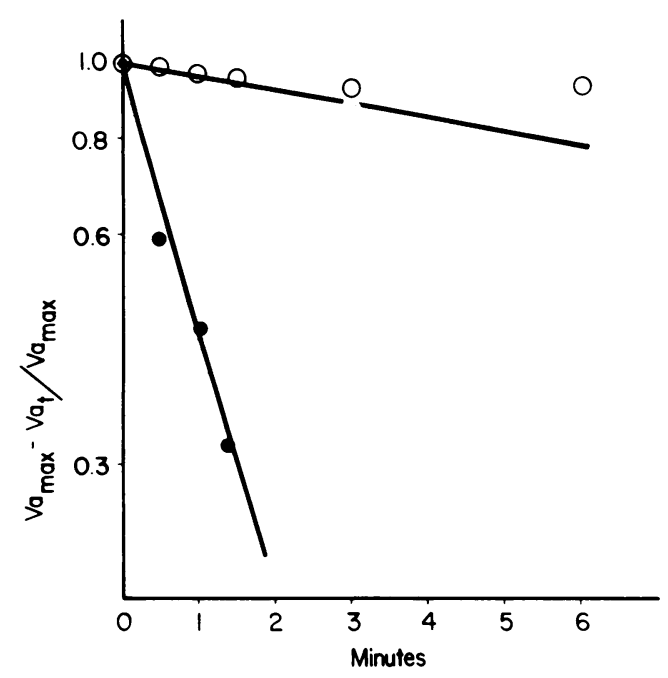

FIGURE 2 Relative rates of reaction of thrombin and thrombin Quick with Factor V. The fraction of Factor $V$ remaining, determined by appearance of Factor $\mathrm{Va}$, is plotted on the ordinate and the activation time is shown on the abscissa. $\mathrm{Va}_{\max }$, maximal $\mathrm{Va}$ activity; $\mathrm{Va}_{\mathrm{t}}$, $\mathrm{Va}$ activity at time, $\mathrm{t}$. The rates have been normalized to equivalent protein concentrations for thrombin and thrombin Quick and the initial four points were used to determine the relative rates of activation. The reaction mixtures contained $0.25 \mathrm{mg} / \mathrm{ml}$ Factor $\mathrm{V}$ and $0.56 \mu \mathrm{g} / \mathrm{ml}$ thrombin (๑) or $2.6 \mu \mathrm{g} / \mathrm{ml}$ thrombin Quick (O) in $0.15 \mathrm{M} \mathrm{NaCl}$, $0.02 \mathrm{M}$ imidazole, $\mathrm{pH} 7.4$ at $22^{\circ} \mathrm{C}$. At the indicated times, aliquots of the reaction mixture were diluted $1 / 200$ into cold assay buffer and subsequently further diluted for assay.
Dodecyl sulfate gel electrophoresis of Factor $\mathrm{V}$ after activation by thrombin and thrombin Quick showed the same fragmentation pattern with both enzymes (data not shown). For Factor VIII activation, the quantitative interpretation is complicated by the rapid loss of Factor VIII activity after activation. However, thrombin Quick at a concentration about 20 times that of thrombin yielded comparable activation of Factor VIII.

In addition to the plasma coagulation factors, platelets are an essential component of the normal hemostatic mechanism and their aggregation may be induced by thrombin. Both thrombin and thrombin Quick induced aggregation of gel-filtered platelets. At an enzyme concentration of $0.07 \mu \mathrm{g} / \mathrm{ml}$, where rapid and complete aggregation was observed with thrombin, no aggregation was observed with thrombin Quick. Based on the amount of protein required to give an equivalent lag time from thrombin addition to initiation of platelet aggregation, thrombin Quick was about $1 / 50$ as effective as normal thrombin.

Several protein proteinase inhibitors play important roles in the regulation of hemostasis. These include the protein designated antithrombin III. The interaction of thrombin and thrombin Quick with antithrombin III was examined to determine whether complex formation occurred. Results of dodecyl sulfate gel electrophoresis are shown in Fig. 3. A stable, presumably covalent, complex was formed between thrombin Quick and antithrombin III and significantly less degradation of this complex occurred compared to that

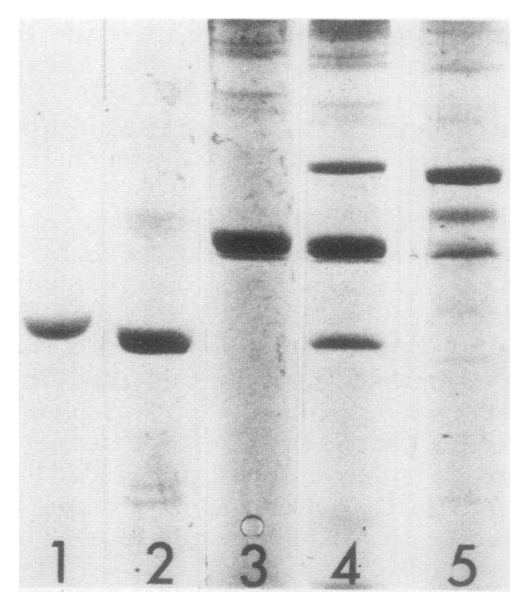

Figure 3 At III complex formation with thrombin or thrombin Quick. Dodecyl sulfate gel electrophoresis of (1) thrombin Quick, $2 \mu \mathrm{g}$; (2) thrombin, $3.5 \mu \mathrm{g}$; (3) Antithrombin III, $12 \mu \mathrm{g}$; and (4) thrombin Quick, $2 \mu \mathrm{g}$, plus antithrombin III, $6 \mu \mathrm{g}$, and (5) thrombin, $2 \mu \mathrm{g}$ plus antithrombin III, $6 \mu \mathrm{g}$. For gels 4 and 5 , the proteins were mixed and incubated 8 min before adding sodium dodecyl sulfate. Protein concentrations in the incubation mixtures were (4) $0.02 \mathrm{mg} / \mathrm{ml}$ thrombin Quick, $0.061 \mathrm{mg} / \mathrm{ml}$ antithrombin III and (5) $0.17 \mathrm{mg} / \mathrm{ml}$ thrombin and $0.64 \mathrm{mg} / \mathrm{ml}$ Antithrombin III. 
with the normal thrombin complex, as might be anticipated from the decreased activity of thrombin Quick observed in the above reactions. Analysis of this reaction by scanning densitometry of gels run after incubation of thrombin Quick $(0.028 \mathrm{mg} / \mathrm{ml})$ with antithrombin III $(0.16 \mathrm{mg} / \mathrm{ml})$ showed that $35 \%$ of the thrombin Quick was converted to complex at $10 \mathrm{~min}$. Prolonged $(1 \mathrm{~h})$ incubation or addition of heparin $(1 \mu \mathrm{g} / \mathrm{ml})$ increased the extent of reaction of thrombin Quick to only $50 \%$. Thus a complex between thrombin Quick and antithrombin III is readily formed, but under conditions where the reaction between thrombin and antithrombin III goes to completion, only half of the thrombin Quick preparation forms a complex. A summary of results comparing thrombin and thrombin Quick is shown in Table I.

The observation that the reaction between thrombin Quick and antithrombin III does not reach completion raises questions regarding the integrity of thrombin Quick. Therefore, the thrombin Quick active site was titrated with DAPA, a fluorescent probe known to compete with substrates for the thrombin active site. DAPA binding to thrombin results in fluorescent enhancement, and the $K_{d}$ for this binding is reported as $43 \mathrm{nM}$ (26). Because of the low dissociation constant DAPA may be used as an active site titrant. In parallel reactions thrombin and thrombin Quick were titrated with DAPA and the emission at $550 \mathrm{~nm}$ was determined after excitation at both 340 and $290 \mathrm{~nm}$. The results are shown in Fig. 4. The results obtained from these titrations, summarized in Table II, indicate that, based on equivalent extinction coefficients for thrombin and thrombin Quick, only 35-40\% of the thrombin Quick molecules possess sites titratable with DAPA to yield fluorescence enhancement. The binding affinity is comparable for both enzymes, and similar to that reported for thrombin (26). It has been observed that energy transfer from protein to DAPA occurs as evidenced by DAPA fluorescence emission in response to excitation at the protein absorption maximum. This energy transfer results in a greater fluorescence enhancement than that seen with excitation at the

TABLE I

Comparison of Activity of Thrombin Quick with Thrombin*

\begin{tabular}{lc}
\hline \multicolumn{1}{c}{ Reaction } & $\begin{array}{c}\text { Activity thrombin Quick/ } \\
\text { activity thrombin }\end{array}$ \\
\hline Factor V activation & 0.05 \\
Factor VIII activation & 0.05 \\
Platelet aggregation & 0.02 \\
Fibrinogen & 0.005 \\
Antithrombin III & complex formed \\
\hline
\end{tabular}

\footnotetext{
* Based on total protein as determined by $A_{280}$, assuming the same extinction coefficients.
}
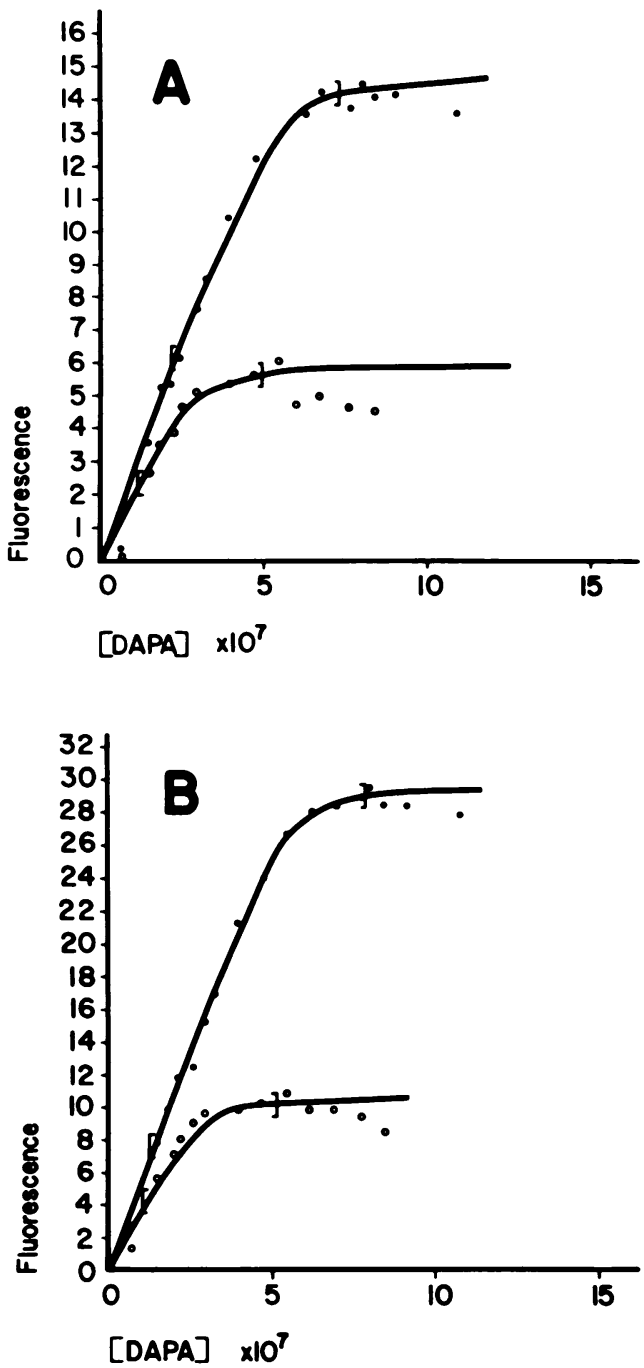

FIGURE 4 Titration of thrombin Quick (0.637 $\mu \mathrm{M}, \mathrm{O})$ and thrombin $(0.659 \mu \mathrm{M}, \bigcirc)$ with the competitive inhibitor, DAPA. Fluorescence was determined at $550 \mathrm{~nm}$. Excitation at (A) 340 and (B) $290 \mathrm{~nm}$. Solid lines are calculated binding curves for the parameters given in Table II, which were derived from the data enclosed within the brackets for each titration (Methods).

DAPA absorption maximum, ${ }^{2}$ a finding which is confirmed in this study. It is also observed that the fluorescence per active site titrated is similar for thrombin and thrombin Quick. The reason for the decrease in fluorescence observed at higher DAPA concentrations is not clear, but suggests a quenching phenomenon due to DAPA protein interaction since the free DAPA fluorescence is subtracted to yield the titration curves presented in Fig. 4.

${ }^{2}$ Straight, D. L., M. E. Nesheim, K. G. Mann, and R. L. Lundbland. Manuscript in preparation. 
TABLE II

Titration of Thrombin and Thrombin Quick with DAPA*

\begin{tabular}{lcccc}
\hline & Excitation & $\mathrm{n}$ & $K_{\mathrm{d}}$ & $\mathrm{F} / \mathrm{nt}$ \\
\hline \multirow{4}{*}{ Thrombin } & $n m$ & $m o l$ & $n m$ & \\
& 290 & 0.83 & 4.6 & 5.4 \\
Thrombin Quick & 340 & 0.83 & 10 & 2.7 \\
& 290 & 0.35 & 15 & 4.9 \\
& 340 & 0.41 & 12 & 2.2 \\
\hline
\end{tabular}

* For procedures see Methods. n, mole fraction of active sites; $K_{\mathrm{d}}$, molar dissociation constant; $F$, maximum fluorescence obtained from the iteration procedure used to determine $n$ and $K_{\mathrm{d}}$. The units are arbitrary based on relative fluorescence.

\section{DISCUSSION}

The earlier finding that the functional prothrombin assayed in the plasma containing prothrombin Quick was $<2 \%$ of normal, whereas the immunologic level of prothrombin was $34 \%$ of normal might be explained by a defect in the activation peptide, a defect at a Factor Xa cleavage site or by the production of a dysfunctional thrombin. It was found that prothrombin Quick was adsorbed to barium citrate (6), which indicates that the $\mathrm{Ca}^{2+}$ and lipid-binding functions of the fragment 1 region are normal (28). Since prothrombin Quick is fragmented normally on activation by known prothrombin activators, and the resultant enzyme has the dodecyl sulfate electrophoretic properties of thrombin, the presence of a dysthrombin, thrombin Quick is indicated. This is consistent with the earlier observation that both one and two stage assays for prothrombin in the dysprothrominemic plasma yielded similarly low levels of prothrombin.

In this study, the activity of thrombin Quick has been compared to that of thrombin using the absorbance at $280 \mathrm{~nm}$ to establish relative concentrations of the proteins. On this basis, thrombin Quick possesses only 0.4 the number of active sites found with thrombin as shown by the titration with DAPA. This finding is confirmed by the observation that only half of the thrombin Quick preparation reacts with antithrombin III. Although a portion of the thrombin Quick preparation appears to be inactive by these criteria, the preparation behaves as a homogeneous protein identical to thrombin by the physical criterion of dodecyl sulfate gel electrophoresis, either reduced or unreduced. It is not possible to ascertain from these results whether the apparently inactive portion of thrombin Quick is totally lacking an active site. It may bind DAPA, but without fluorescence enhancement or energy transfer. Examination of the results presented in Fig. 4A indicates that the affinity of such binding, if present, must be lower by at least a factor of 100 .
Several possible explanations for the observed behavior may be suggested. Thrombin Quick may be a less stable protein than thrombin and consequently subject to partial denaturation and loss of the DAPA binding site to the extent indicated by the titration results. Evidence against this is the observed stability of thrombin Quick preparations stored at $-70^{\circ}$. DAPA titration results similar to those presented were obtained on the same preparation one month earlier. Another possibility is that the thrombin Quick preparation is highly contaminated with prethrombin 2 . This explanation is excluded by the previously mentioned coelectrophoresis of reduced thrombin and thrombin Quick since prethrombin 2 is a single polypeptide chain that is hydrolyzed to form the two chains of thrombin. No prethrombin 2 is seen on the reduced gels.

Human thrombin is known to differ from bovine thrombin by the absence of an homologous 13 amino acid peptide at the amino terminal end of the $A$ chain. This peptide is removed from human thrombin autocatalytically (29), and because of this, the effect of the peptide on thrombin activity is not known. For thrombin Quick, the decreased activity may result in slow cleavage of this peptide and affect the observed activity of the thrombin Quick preparation. However, electrophoresis results, as well as the stability of the preparation discussed above suggest that the hydrolysis of the amino terminal peptide is complete. Other explanations of the active site titration results include the substitution of an Arg residue at some point in the A chain of thrombin such that a slow cleavage of the A chain occurs autocatalytically with resultant alterations in the titration behavior with DAPA. Such an alteration in the A chain would not be detected by electrophoresis. Because of the low molecular weight of the A chain, only the B chain of thrombin is observed on dodecylsulfate gel electrophoresis in the presence of reducing agents.

Another explanation for the apparently altered number of active sites is that there are two dysthrombins in the preparation. This could arise if the patient were heterozygous for two dysthrombins, although the finding of two in one individual should be a very rare occurrence. However, this now appears to be a possibility in this patient. The presence of two dysthrombins, one possessing an active site titrated by DAPA and the other inactive by this criterion would explain the results of both the DAPA titration and the reaction with antithrombin III. Further investigation of this possibility continues and will include an attempt to identify two precursor dysprothrombins. It has not been possible to clearly establish the mode of inheritance in this patient. The patient's mother is deceased; however plasma from her father shows $41 \%$ prothrombin in one stage taipan venom assay and $104 \%$ prothrombin by immunologic assay (unpublished observa- 
tion) suggesting that he is heterozygous for normal prothrombin and a dysprothrombin. It is possible that she could have also inherited a dysprothrombin from her mother; her siblings have not been available for study.

The results of the DAPA titration indicate that for the titratable molecules, the active site environment in thrombin Quick retains certain similarity to that in thrombin as evidenced by similar dissociation constants for the titrant, similar fluorescent enhancement determined by excitation at both 340 and $290 \mathrm{~nm}$ and consequently a similar active site geometry to the extent required to facilitate energy transfer from the protein to DAPA. The defect in thrombin Quick, as evidenced by low activity with biological substrates must lie in some other aspect of the active site. In this regard it may be noted that both alpha and beta thrombins behave similarly with respect to DAPA binding while having differing catalytic behavior (26). The notion that thrombin Quick activity may represent only a thrombin contaminant is discounted by the differing behavior of thrombin Quick relative to thrombin in the various reactions studied. The relatively low activity observed in thrombin-catalyzed reactions with Factors $\mathrm{V}$ and VIII, fibrinogen, and platelets contrasts remarkably with the observed ease of formation of a complex with antithrombin III and the presence of DAPA binding sites.

Even if only one-third of thrombin Quick molecules have active sites, the activity of thrombin Quick on Factors V and VIII, and platelets, is no more than $15 \%$ that of thrombin and seems to occur much less readily than antithrombin III complex formation. An even greater defect $(\sim 1 \%)$ is seen with fibrinogen. It is hoped that this difference may be exploited further to investigate what must be a fundamental difference in reaction mechanisms for these various events in normal hemostasis.

The identification of a dysthrombin provides a unique opportunity for investigation of the mechanism of thrombin's action. If it is assumed that a defective thrombin is due to a single point mutation and consequently a single amino acid replacement in the primary structure, a thorough understanding of the properties of the resultant thrombin as well as identification of the primary structure defect should allow an increased understanding of structure-function relationships in the enzyme thrombin.

\section{ACKNOWLEDGMENTS}

The authors would like to thank Mr. Richard D. Crowley and Ms. Catherine A. Mikus for assistance in plasma collection. We are also indebted to V.A. for supplying plasma and to Ms. Rhonda Demuth for typing the manuscript.

\section{REFERENCES}

1. Shapiro, S. S., J. Martinez, and R. R. Holburn. 1969. Congenital Dysprothrombinemia: an inherited structural dis- order of human prothrombin. J. Clin. Invest. 48: 22512259.

2. Josso, F., J. M. de Sanchez, J. M. Lavergne, D. Menache, and J. P. Soulier. 1971. Congenital abnormality of the prothrombin molecule (factor II) in four siblings: prothrombin Barcelona. Blood. 38: 9-16.

3. Shapiro, S. S., N. Maldonado, and J. Fradera. 1973. Prothrombin San Juan: a third congenital dysprothrombinemia. In IVth International Congress on Thrombosis and Haemostasis: Abstracts, Vienna, International Society on Thrombosis and Haemostasis. 262. (Abstr.)

4. Kahn, M. J. P., and A. Govareris. 1974. Prothrombin Brussels, a new congenital defective protein. Thromb. Res. 5: 141-156.

5. Girolami, A., G. Bareggi, A. Burnetti, and A. Sticchi. 1974. Prothrombin padua: a "new" congenital dysprothrombinemia. J. Lab. Clin. Med. 84: 654-666.

6. Owen, C. A., R. A. Henriksen, F. C. McDuffie, and K. G. Mann. 1978. Prothrombin Quick, a newly identified dysprothrombinemia. Mayo Clin. Proc. 53: 29-33.

7. Girolami, A., S. Coccheri, G. Palareti, M. Poggi, A. Burul, and G. Capellato. 1978. Prothrombin molise: a "new" congenital dysprothrombinemia, double heterozygosis with an abnormal prothrombin and "true" prothrombin deficiency. Blood. 52: 115-125.

8. Bezeaud, A., M. C. Guillin, D. Menache, F. Olmeda, M. Quintan, and N. Gomez. 1978. Prothrombin madrid: a new familial abnormality of prothrombin. In Abstracts International Society of Hematology, Paris, France. 424.

9. Rabiet, M. J., J. Elion, D. Labie, and F. Josso. 1979. Prothrombin metz: purification and characterization of a variant of human prothrombin. Thromb. Haemostasis. 42: 57. (Abstr. 124)

10. Davie, E. W., K. Fujikawa, K. Kurachi, and W. Kisiel. 1979. The role of serine proteases in the blood coagulation cascade. Adv. Enzymol. Relat. Areas Mol. Biol. 48: 277-318.

11. Jackson, C. M., C. T. Esmon, S. N. Gitel, W. G. Owen, and R. A. Henriksen. 1975. The conversion of prothrombin to thrombin: the function of the propiece of prothrombin. In, Prothrombin and Related Coagulation Factors. H. C. Hemker and J. J. Veltkamp, editors. Leiden University Press, The Hague, Netherlands. 59-88.

12. Quick, A. J., A. V. Pisciotta, and C. V. Hussey. 1955. Congenital hypoprothrombinemic states. Arch. Intern. Med. 95: 2-14.

13. Quick, A. J. 1974. The Hemorrhagic Diseases and the Pathology of Hemostasis. Charles C Thomas, Springfield, Ill. 229-235.

14. Aronson, D. L. 1966. Chromatographic differentiation of human prothrombins. Thrombos. Diath. Haemorrh. 16: 491-496.

15. Lundblad, R. L. 1971. A rapid method for the purification of bovine thrombin and the inhibition of the purified enzyme with phenylmethylsulfonyl fluoride. Biochemistry. 10: 2501-2506.

16. Kézdy, F. J., L. Lorand, and K. D. Miller. 1965. Titration of active centers in thrombin solutions. Standardization of the enzyme. Biochemistry. 4: 2302-2308.

17. Laemmli, U. K. 1970. Cleavage of Structural Proteins during the Assembly of the Head of Bacteriophage T4. Nature (Lond.). 227: 680-685.

18. Nesheim, M. E., K. H. Myrmel, L. Hibbard, and K. G. Mann. 1979. Isolation and characterization of Single Chain Bovine Factor V. J. Biol. Chem. 254: 508-517.

19. Wagner, R. H., W. D. McLester, M. Smith, and K. M. Brinkhous. 1964. Purification of antihemophilic factor 
(Factor VIII) by amino acid precipitation. Thromb. Diath. Haemorrh. 11: 64-74.

20. Hardisty, R. M., and J. C. MacPherson. 1962. A one-stage Factor VIII (antihemophilic globulin) assay and its use on venous and capillary plasma. Thromb. Diath. Haemorrh. 7: 215-229.

21. Tangen, O., and H. J. Berman. 1972. Gel Filtration of Blood Platelets: A Methodological Report. Adv. Exp. Med. Biol. 34: 235-243.

22. Lages, B., M. C. Scrutton, and H. Holmsen. 1975. Studies on gel-filtered platelets: isolation and characterization in a medium containing no Added $\mathrm{Ca}^{2+}, \mathrm{Mg}^{2+}$, or $\mathrm{K}^{+}$. J. Lab. Clin. Med. 85: 811-825.

23. Born, G. V. R., and M. J. Cross. 1963. The aggregation of blood platelets. J. Physiol. 168: 178-195.

24. Straughn, W., III, and R. H. Wagner. 1966. A simple method for preparing fibrinogen. Thromb. Diath. Haemorrh. 16: 198-206.

25. Owen, W. G. 1975. Evidence for the formation of an ester between thrombin and heparin cofactor. Biochim. Biophys. Acta. 405: 380-\$87.

26. Nesheim, M. E., F. G. Prendergast, and K. G. Mann. 1979. Interactions of a fluorescent active-site-directed inhibitor of thrombin: dansylarginine $\mathrm{N}$-(3-ethyl-1,5-pentanediyl)amide. Biochemistry. 18: 996- 1003.

27. Gutfreund, H. 1972. Enzymes: Physical Principles. John Wiley \& Sons Ltd., Sussex, England. 68-72.

28. Gitel, S. N., W. G. Owen, C. T. Esmon, and C. M. Jackson. 1973. A polypeptide region of bovine prothrombin specific for binding to phospholipids. Proc. Natl. Acad. Sci. U. S. A. 70: 1344-1348.

29. Downing, M. R., R. J. Butkowski, M. M. Clark, and K. G. Mann. 1975. Human prothrombin activation. J. Biol. Chem. 250: 8897-8906.

30. Jackson, C. M., R. A. Henriksen, C. W. Peng, and E. T. Yin. 1976. Report of the task force on preparation of factor Xa (bovine) PRP (ICTHR). Thromb. Haemostasis. 35: 479-482. 